\title{
Status of lung cancer screening
}

\author{
James L. Mulshine \\ Internal Medicine, Rush University, Chicago, IL 60612, USA \\ Correspondence to: Professor James L. Mulshine, MD. Internal Medicine, Rush University, Chicago, IL 60612, USA. \\ Email: james_1_mulshine@rush.edu. \\ Provenance: This is an invited Editorial commissioned by Executive Editor-in-Chief Dr. Jianxing He (Department of Cardiothoracic Surgery, The \\ First Affiliated Hospital of Guangzhou Medical University, Guangzhou, China). \\ Comment on: Chung M, Tam K, Wallace C, et al. International Early Lung Cancer Action Program: update on lung cancer screening and the \\ management of CT screen-detected findings. AME Med J 2017;2:129.
}

Submitted Oct 12, 2017. Accepted for publication Oct 16, 2017.

doi: $10.21037 /$ jtd.2017.10.121

View this article at: http://dx.doi.org/10.21037/jtd.2017.10.121

CT screening emerged as an approach to improve the curability of asymptomatic lung cancer as reported in a landmark study by Henschke and her multi-disciplinary colleagues in 1999 (1). Now eighteen years later, we have a follow-up report from this group on the status of low dose CT screening particularly focusing on the screening management process in optimally delivering this service. In the years since their initial screening report, this group has developed a unique confederated structure called the International-Early Lung Cancer Action Project (I-ELCAP) to advance research while delivering best-practice with the screening process (2). This approach was adopted so that collaborating screening researchers could pool resources from their respective institutions to more rapidly complete research around the many aspects of lung cancer screening. This approach is a pragmatic accommodation to the nature of lung cancer screening, even in a defined high risk for lung cancer cohort, which will involve finding lung cancer cases typically with a frequency of about $1 \%$ on baseline. To have sufficient numbers of informative cases to conduct significant research in a timely fashion requires a vast screening populations and so cooperative interactions of many institutions pooling accrual cases is one way to address this challenge. The consortium of institutions comprising the I-ELCAP has now published a large range of studies on aspects of lung cancer screening management. A recent review was a status report of I-ELCAP current activities and it is a distillation of their body of work, organized by a series of questions that span the topic of screening, beginning with who should be screened (3).
As the benefit of screening emerges through a balance of potential harms and benefits actually experienced by an individual in a screening process, the answer to this "who should be screened question" is complex. The authors review the rationale for the United States Preventative Service Task Force recommendation but also point out that this is derived from the results of the National Lung Screening trial $(4,5)$. The challenge inherent in this issue is that the National Lung Screening Trial was designed to evaluate if there was a significant benefit from low dose CT screening in a high-risk tobacco exposed population. A trial design was selected for that purpose and not to address the spectrum of individuals with variable tobacco and or occupational exposures who may also benefit from participation in a lung cancer screening program. This tension highlights an important public health challenge. Screening trials tend to be large and expensive both in dollars and well as in time consumption required to conduct and analyze such studies. For this reason, a number of investigators have explored the use of analytic models to tailor screening recommendations in a more efficient way to address the transition of screening from a validating trial into a clinical practice. As reviewed by the authors, a large number of studies are emerging around risk prediction models but as these modeling studies are new, it is difficult to validate that their findings are truly robust. This is a critical avenue for further scrutiny as there are many research studies being published that may have an impact on the perceived value proposition within the screening setting for individuals deciding on participating in this new 
preventative service (3).

The authors also explore the question regarding the optimal frequency of repeat rounds screening since lung cancer risk is continuous in smokers and persists in former smokers. First, they consider the screening frequency in regard to the health care cost of the service. More frequent screening can aid in the sensitivity of the service and correspondingly to the frequency of early stage cancer detection but there are economic costs related to delivering the screening service. Conversely, if the screening interval is lengthened as reported by the Dutch/Belgian screening trial group, NELSON, the frequency of symptomdetected cancers found within the interval of screening rises (6). Symptom-detected cancers are much more likely to be advanced, non-curable lung cancers, which represents a failure of the screening process. In addition, advanced cancer in addition is more expensive to care for compared to early stage lung cancer. Therefore, the current recommendations in the United States are for annual CT screening but clearly this also is an area where further research would be helpful to maximize the yield of curative, screen-detected lung cancers in the most efficient fashion across the complex array of at-risk for lung cancer cohorts.

Next, the authors explore how to optimally detect suspicious pulmonary nodules in the process of screening. Many of the definitions of X-ray findings in the screening setting involved terms that I-ELCAP investigators first developed and now these terms are used by the screening community across the world. In addition, I-ELCAP research was critical in defining the natural history of many of these new types of pulmonary lesions found in this screening setting. Further, I-ELCAP research publications have been critical to this process and complemented by a significant effort of the International Association for the Study of Lung Cancer work with the pathology and staging of this early lung findings (7). Such that clinical management in the screening setting is guided by the aggressiveness of the specific lesion and this has allowed for an improved calibration of surgical management to avoid over-treatment.

In this work, this issue of how to accurately characterize nodule size has emerged as a critical determinant for clinical management (8). In these determinations, standard manual two dimensional measurements are the dominant clinical process, but the author point out that computerbased assessments of volume are likely to come forward. The advantage of volumetric nodule determination may be due to greater accuracy. This becomes especially important when the growth rate of small nodules is measured by looking at the change in volume measurements across a defined time interval. This allows for a calculation of doubling-time, which has been reported to be an important imaging biomarker reflecting clinical aggressiveness (9). The authors discuss how nodule size and doubling times can be used to improve the personalization of the screening management process.

The measurement of nodule volume and its use as an imaging biomarker has been the focus of an ongoing collaboration with the Radiology Society of North America's Quantitative Biomarker Alliance (QIBA) and the I-ELCAP (10,11). A recent publication describes how the process of volume measurement can be standardized to ensure optimal accuracy of the volume determinations (12). Quantitative imaging is an important new area for radiology, and measuring small pulmonary nodules in the range of $5-12 \mathrm{~mm}^{3}$ with sufficient precision to define a $95 \%$ confidence interval for the determination is a particularly challenging task. However, precise measurements may allow for responsible clinical decision making within relatively brief time intervals by using a standardized quantitative image data acquisition approaches as established by QIBA. Dissemination of the quantitative imaging acquisition and quality approaches can potentially be accomplished by leveraging cloud-based computing environment as it can allow easy access to defined automated image quality analyses using integrated reference tools to evaluate conformance with best quantitative imaging practice (13).

As suggested by the review, these quantitative approaches for lung cancer screening may also allow for evaluation of other tobacco-related thoracic findings seen on low dose CT scans such as coronary artery calcium deposition and parenchymal lung injury findings consistent with chronic obstructive pulmonary disease as reported by I-ELCAP investigations $(3,14,15)$. The simultaneous analysis of the three tobacco-related diseases in a defined high-risk population that also happen include individuals at-risk for three of the most lethal diseases in most societies, is an extraordinary public health opportunity. Research to sort out the complex interactions across these three complex diseases is already underway with researchers from I-ELCAP as well as NELSON. In the review, it was pointed out that $85 \%$ of smokers do not get lung cancer (3). Early research on outcomes of low dose CT lung cancer screened individuals suggests that while only $1 \%$ of screening subjects may have lung cancer on baseline screening. However, more than $20 \%$ of CT screening candidates may 
have evidence of clinically significant coronary calcium and upwards of $25 \%$ may have objective evidence of chronic obstructive pulmonary disease (COPD) $(14,15)$. These thoracic cavity confined comorbidities have been extensively characterized in the low-dose CT literature and integrating screening of heavily tobacco-exposed cohorts with best practice tobacco cessation approaches has been shown to enhance the cost utility of low-dose CT screening (16). In addition, clinical interventions have already been developed by the cardiology community as the implications of coronary calcium status are well established (17).

Analysis of quantitative imaging data with low dose CT screening may provide remarkably productive biomarker tools to help clinicians discriminate the individual risk of a heavily tobacco exposed subjects enrolled in the screening process to enable a more targeted approach to recommending intensive tobacco cessation, diet, exercise and other lifestyle interventions as well a specific new, precision medicine agents to efficiently and economically improve health outcomes. A remarkable amount of this research has emerged from the I-ELCAP group and their many collaborators. Their CT screening findings have now been validated by many other research groups. The challenge now is to efficiently communicate this new and rapidly evolving body of information to the already busy, primary care community so we can ensure that relevant individuals can get access to this important new detection service (18).

\section{Acknowledgements}

None.

\section{Footnote}

Conflicts of Interest: The author has no conflicts of interest to declare.

\section{References}

1. Henschke CI, McCauley DI, Yankelevitz DF, et al. Early Lung Cancer Action Project: overall design and findings from baseline screening. Lancet 1999;354:99-105.

2. Henschke CI, Yankelevitz DF, Smith JP, et al. Screening for lung cancer: the early lung cancer action approach. Lung Cancer 2002;35:143-8.

3. Chung M, Tam K, Wallace C, et al. International Early Lung Cancer Action Program: update on lung cancer screening and the management of CT screen-detected findings. AME Med J 2017;2:129.

4. Moyer VA, U.S. Preventive Services Task Force. Screening for lung cancer: U.S. Preventive Services Task Force recommendation statement. Ann Intern Med 2014;160:330-8.

5. National Lung Screening Trial Research Team, Aberle DR, Berg CD, et al. The National Lung Screening Trial: overview and study design. Radiology 2011;258:243-53.

6. Horeweg N, Scholten ET, de Jong PA, et al. Detection of lung cancer through low-dose CT screening (NELSON): a prespecified analysis of screening test performance and interval cancers. Lancet Oncol 2014;15:1342-50.

7. Field JK, Smith RA, Aberle DR, et al. International association for the study of lung cancer computed tomography screening workshop 2011 report. J Thorac Oncol 2012;7:10-9.

8. Henschke CI, Yip R, Yankelevitz DF, et al. Definition of a positive test result in computed tomography screening for lung cancer: a cohort study. Ann Intern Med 2013;158:246-52.

9. Yankelevitz DF, Reeves AP, Kostis WJ, et al. Small pulmonary nodules: volumetrically determined growth rates based on CT evaluation. Radiology 2000;217:251-6.

10. Ma X, Siegelman J, Paik DS, et al. Volumes learned: it takes more than size to "Size Up" pulmonary lesions. Acad Radiol 2016;23:1190-8.

11. Mulshine JL, Gierada DS, Armato SG 3rd, et al. Role of the quantitative imaging biomarker alliance in optimizing CT for the evaluation of lung cancer screen-detected nodules. J Am Coll Radiol 2015;12:390-5.

12. Rydzak CE, Armato SG, Avila RS, et al. Quality assurance and quantitative imaging biomarkers in low dose CT lung cancer Screening. Br J Radiol 2017. [Epub ahead of print].

13. Sevick-Muraca EM, Frank RA, Giger ML, et al. Moonshot acceleration factor: medical imaging (Meeting Report). Cancer Res October 9 2017. Available online: http://cancerres.aacrjournals.org/content/ early/2017/10/08/0008-5472.CAN-17-1698.full-text.pdf

14. Htwe Y, Cham MD, Henschke CI, et al. Coronary artery calcification on low-dose computed tomography: comparison of Agatston and Ordinal Scores. Clin Imaging 2015;39:799-802.

15. Zulueta JJ. Emphysema and Lung Cancer. More than a coincidence. Ann Am Thorac Soc 2015;12:1120-1.

16. Villanti AC, Jiang Y, Abrams DB, et al. A cost-utility analysis of lung cancer screening and the additional benefits of incorporating smoking cessation interventions. 
PLoS One 2013;8:e71379.

17. Gepner AD, Young R, Delaney JA, et al. Comparison of coronary artery calcium presence, carotid plaque presence, and carotid intima-media thickness for cardiovascular disease prediction in the Multi-Ethnic
Study of Atherosclerosis. Circ Cardiovasc Imaging 2015;8. pii: e002262.

18. Mulshine JL, D'Amico TA. Issues with implementing a high-quality lung cancer screening program. CA Cancer J Clin 2014;64:352-63.

Cite this article as: Mulshine JL. Status of lung cancer screening. J Thorac Dis 2017;9(11):4311-4314. doi: 10.21037/ jtd.2017.10.121 